\title{
Are Forum Networks Social Networks? A Methodological PeRspective
}

\author{
Oleksandra Poquet \\ Centre for Change and Complexity in Learning (C3L) \\ University of South Australia \\ Adelaide, SA, Australia \\ sspoquet@gmail.com
}

\author{
Liubov Tupikina \\ Center for Research and Interdisciplinarity (CRI) \\ Universite de Paris \\ Bell Labs, Nokia \\ Paris, France \\ liubov.tupikina@cri-paris.org
}

\author{
Marc Santolini \\ Center for Research and Interdisciplinarity (CRI) \\ Universite de Paris \\ Center for Complex Network Research, Northeastern University \\ Paris, France \\ marc. santolini@cri-paris.org
}

December 27, 2019

\begin{abstract}
The mission of learning analytics (LA) is to improve learner experiences using the insights from digitally collected learner data. While some areas of LA are maturing, this is not consistent across all LA specialisations. For instance, LA for social learning lack validated approaches to account for the effects of cross-course variability in learner behavior. Although the associations between network structure and learning outcomes have been examined in the context of online forums, it remains unclear whether such associations represent bona fide social effects, or merely reflect heterogeneity in individual posting behavior, leading to seemingly complex but artefactual social network structures. We argue that to start addressing this issue, posting activity should be explicitly included and modelled in forum network representations. To gain insight to what extent learner degree and edge weight are merely derivatives of learner activity, we construct random models that control for the level of posting and post properties, such as popularity and thread hierarchy level. Analysis of forum networks in twenty online courses presented in this paper demonstrates that individual posting behavior is highly predictive of both the breadth (degree) and frequency (strength) in forum communication networks. This implies that, in the context of forum-based modelling, degree and frequency may not reflect the social dynamics. However, results suggest that clustering of the network structure is not a derivative of individual posting behaviour. Hence, weighted local clustering coefficient may be a better proxy for social relationships. The empirical results are relevant to scientists interested in social interactions and learner networks in digital learning, and more generally to researchers interested in deriving informative social network models from online forums.
\end{abstract}

Keywords null models, online forums, online learning, social networks

\section{Introduction}

Learning analytics (LA) strives to improve learner experiences using insights from digital learner traces. Recently LA has been criticized for its limited impact on the educational practices and restricted contributions to learning theories [1, 2]. The field's focus on exploratory course-based studies in lieu of larger evaluations has diminished the broader impact of LA [1]. Despite the push for large-scale evaluations of learning, some research areas of LA remain relatively 
immature and lack the tools and approaches necessary for scaling and systematic replication. Computational analyses of social interactions in digital learning environments is such an area.

LA researchers have been interested in social online interactions since the first Learning Analytics and Knowledge conference in 2011 (LAK'11). Capturing learner interactions as network representations helps visualize social dynamics in online course forums making them a popular element in LA dashboards [3, 4]. Social network analysis (SNA) then enables to quantify social interactions and examine measures of network centrality in course forums against learning outcomes [5]. As a result, SNA has been applied in a range of contexts: from university online courses [6] and MOOCs [7, 8], to social text- and video-annotation scenarios [9], as well as informal learning settings [9].

Despite its uptake across a broad range of learning contexts, the so-called social learning analytics remains relatively immature. Currently, analyses of forum networks lack rigorous evaluation strategies that can help interpret individual and network-level measures of social interactions. Multiple approaches to examining course-level forum networks have been suggested. For instance, researchers have examined the association between academic performance with descriptive network-level (e.g. density) [10] and node-level (i.e. degree) measures in online forums [5]. Alternatively, within individual courses, descriptive statistics are used to show that forum networks increase in density, as well as in reciprocal and transitive patterns throughout the course [11, 12]. It has also been suggested that network structure mediates the association of network centrality and academic performance [13]. However, little work has been done to examine if these observed patterns occur by chance or result from a specific socio-technical or pedagogical intervention.

Without the analytical strategies that examine if the observed forum communication has occurred by chance, interpretation of the network patterns is largely limited. Consequently, the thresholds of what is naturally 'high' or 'low' with regards to density, transitivity, centralization, and reciprocity in online learner networks can not be established. The lack of evaluations of forum activity that capture natural and expected dynamics for the formation of forum learner networks presents a significant research gap. Neither learners nor instructors can receive feedback about their actions, given that the observed results may have occurred by chance, and not resulted from specific efforts or instructional intervention. The lack of approaches to compare networks across courses similarly hinders large-scale comparisons of educational interventions necessary for LA's broader impact on learning.

To fill that gap, statistical network modelling can be used to evaluate course-level forum activity. Importantly, modelling of forum interactions in LA thus far has had significant limitations. LA studies have modelled learner-to-learner networks through methodologies like exponential graph modelling (ERGM), stochastic actor-oriented modelling (SAOM), weight randomisation, and similar. Throughout this research, forum networks are often represented and modelled as social networks where forum posting and reply behaviour is transformed into networks of people and direct relationships between them. LA studies go onto examining these networks of people, inquiring if they are formed due to the social processes of reciprocity, transitivity, popularity, and similar social processes. While social processes are fundamental to human networks of friendship, trust, and kinship,forum communication is fundamentally driven by posting activity prompted through course design as well as learner motivations[14]. By excluding posting activity (i.e. learner making a post) from modelling forum networks, the assumptions about what drives communication, besides social dynamics, are not explicit in the modelling and in the interpretation. As a result, such limited examinations of forums can not conclude what the drivers for forum communication were: whether observed patterns of forum communication resulted from individual activity levels, social dynamics (community formation processes), or other exogenous factors (instructor intervention or tool affordance).

In contrast to LA's modelling of forums based on hypotheses about social tie formation, network scientists take a distinctly different approach. As shown in a recent literature review, [15], Internet forums are predominantly represented as networks where posts are nodes: adding a new post is a generative process driving the network formation. To understand the extent to which posting behavior can explain the formation of forum networks in learning settings, this study adopts a null models approach. Null models are graphs simulated to match the observed network in some selected aspects, but otherwise - formed at random. Using forum activity in twenty online courses, we investigate if random networks simulated with fixed individual-level posting activity and post popularity can produce network structures similar to the real-life course forums. We experiment with two varieties of network representations of forum communication: 1) post-tree network and 2) student-to-thread posting network. Results show that even these simple null models can explain both the breadth (degree) and frequency (strength) of forum student networks in a large proportion of the observed forum networks in our dataset. Analyses suggest that other elements of network structure, such as clustering, are not derivatives of individual online posting behaviour and could be more representative of social relationships. 


\section{Literature Review}

\subsection{Posting Activity and Network Centrality in Forums}

Facilitation of learner-to-learner interactions are at the foundation of online teaching and socio-constructivist approaches to learning. Educational researchers emphasize that learner-to-leaner interactions need to be designed for and do not happen naturally [16]. Therefore, for learner-to-learner interactions to effectively impact learning, course design features and forum facilitation strategies need to afford their development. The need to evaluate if designed interactions have been successfully facilitated created an agenda for computational analysis that evaluates forum participation. Thus far, two common approaches to evaluating forum participation are the measures of contributing posts (post count) and creating connections between learners (degree centrality).

The count of posts submitted to the forum is among common approaches to evaluate forum participation. In their early work, Macfadyen and Dawson [17] showed a strong association between the number of discussion replies posted and learner final grade $(\mathrm{r}=.94, \mathrm{p}<.0001)$. The number of posts has been among most common measures of social learning in MOOCs [18], and are commonly included in modelling learner engagement [19] and predicting completion [20].

Indicators of learner positioning in forum communication networks, such as degree centrality, is another approach to quantifying social learning activity. Recently, a number of critiques arose around the validity and generalisability of such networked measures. The approaches to constructing learner-to-learner networks are diverse, resulting in inconsistencies of the associations between learner centralities in these networks and learning outcomes [21, 8]. Further, there is no consensus as to how to construct these networks as well as different ideas as to what these networks may represent.

LA studies of social learning often refer to network positioning measures as a more elaborate way to capture forum participation, beyond the count of posts made by the learner. However, posting activity and networked (interpersonal) activity are strongly related to one another, as learner-to-learner ties in communication networks are derived from learner posting activity. Although learner-to-learner sociograms could be used to represent social processes, they are ultimately projections of post-mediated interactions enabled when learners contribute posts. The so-called co-presence learner networks, where a network tie is defined through shared contributions in the discussion threads, are projections of bipartite relations between learners and discussion threads (here learner relations are mediated by discussion) [22]. The so-called direct post-reply networks are projections of post-to-post activity where a post-to-post relationship is substituted by a learner-to-learner tie. In both scenarios, individual posting activity and the properties of an artefact (posts or discussion) mediate learner-to-learner relationships, on par with the interpersonal processes driving network formation.

\subsection{Limitations of LA Forum Modelling}

The interdependence between posting activity and learner positioning in communication networks draws attention to the number of limitations in forum communication research.

In LA, poster networks rarely include forum posting activity explicitly (as distinct nodes or ties) in network representations and modelling. Instead, researchers construct and model network representations directly as learner-to-learner networks. Statistical network modelling through methods such as exponential random graph models (ERGM) or stochastic actor-oriented models (SAOMs) are used to estimate the processes forming learner-to-learner networks using assumptions about the formation of social networks [23]. Results highlight that reciprocity of interactions often occur beyond chance, whereas closure is less common, and preferential attachment interpreted as learner popularity has not been observed [13, 24, 25].

There are several noteworthy shortcomings of statistical modelling of forums that are solely based on social network hypotheses. First, they model relationships, and not post-mediated/discussion-mediated interactions. That is, they examine forum communication through the hypothesis applied to bona fide social networks through the processes, such as of reciprocity and transitivity. Second, they often embed inconsistent decisions for network construction. Third, they do not control for posting activity: no actor-attribute variable are included to control for the number of posts (instead the extent of learners one interacted with is modelled). Fourth, the number of posts exchanged at the dyadic level is often not captured within the models. ERGMs of forum communication have modelled binary ties between the learners, removing the information about the number of posts shared between two learners from the network modelling process. Similar limitations apply to SAOMs that are not designed to model ties weighted through continuous variables. In light of these shortcomings, the evidence derived from modelling online forum networks to evaluate forum communication activity and learning can be perceived as limited. 
We argue that including posting activity into representations and modelling processes can help understand how communication networks form. Some visualisations of forum activity bring to the fore forum activity at the post-level. Examples include that of Atapattu et al. [26] who visualised clusters of themes comprised of forum posts, and Marbouti et al. [27] who explored visualising posts as a tree-network. A similar emphasis in statistical network modelling is required.

\section{Research Questions}

We have argued that LA lacks evaluations of forum activity that capture natural and expected dynamics for the formation of forum learner networks. Such is a significant research gap as it hinders meaningful interpretations of the observed patterns as well as cross-course comparisons of social learning activity. We suggested that posting activity represents learner behaviour central to forum communication, and should be included in the network representations and statistical modelling.

To better understand the role of posting activity in the formation of forum communication networks, this study examined to what extent individual posting activity plays a role in the formation of forum networks. To unpack this question, we analysed:

i) How much heterogeneity in posting behaviour is observed across the different forum communication networks?

ii) How much of the student network global structure (density, communities) is explained by the online forum posting behaviour?

iii) What individual topological features (local: degree, clustering, leverage centrality, and global: subgraph centrality) are not derivative of online posting behaviour?

\section{Methods}

To understand the extent to which individual posting behavior generates forum networks in learning settings, this study adopts a null models approach (see Section 4.1). We apply two kinds of null models to the dataset of twenty online and blended university courses. Courses were taught by different instructors and utilized different pedagogies for forum participation. Section 4.1 elaborates on the rationale behind using null models. Section 4.2 explains how forum-based modelling approach differs from social modelling that is more commonly adopted in LA. Section 4.3 offers details of randomisation for each of the null models. Section 4.4 provides steps to compare forum networks generated from the null models with the observed forum data.

\subsection{Random Graphs as Null Models}

Null models based on random graph simulations are widely used in network science for hypothesis testing about how networks are formed [28]. Simulations of random graphs, where 'the values of certain [network] properties are fixed, but the network is in other respects random' [29, p. 343], is needed because network data may violate the assumptions of normality and independence of observations. Such random graphs offer a distribution that the observed graph can be compared against.

One common application of null models, i.e. random graphs simulated based on a particular hypothesis about network formation, is towards filtering out 'a true' structure of the network by identifying the strength of links' weights in ego-networks and dyadic networks [28]. That is, random graphs provide a distribution of weights that allows to estimate if the observed weights are significantly different statistically, and in this case, kept as 'true' weights. Similar approach has recently been used to identify significant 'weak' ties [30], extending seminal work from Granovetter [31] around the notion of weak and strong ties to communication networks.

Null models of online forums in learning settings can be simulated with different generative processes related to student activity, contents of the post-messages or discussion threads, post or student popularity, as well as social dynamics of interpersonal relations observed via social selection or social influence processes.

Network science has offered a number of solutions towards statistical modelling of forums that include posting - a practice that can be integrated into LA research. Specifically, network scientists conceptualise posting activity as a fundamental generative process for communication in the Internet forums. Threaded online conversations in reddit or stackoverflow are commonly conceptualised as tree-network structures driven by sequential posting behaviour [32]. In such a tree-network structure, the very first post in the thread is defined as a root, posts are linked with ties if there is an explicit reply-to relationship between them. The depth of the tree represents the depth of the discussion thread. The 


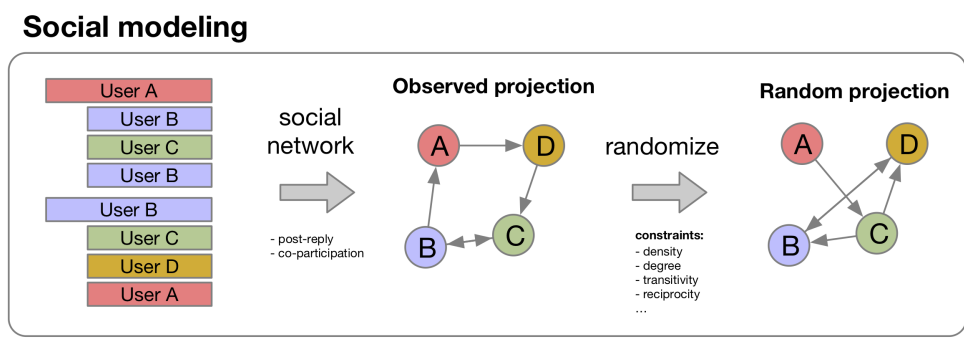

\section{Forum-based modeling}

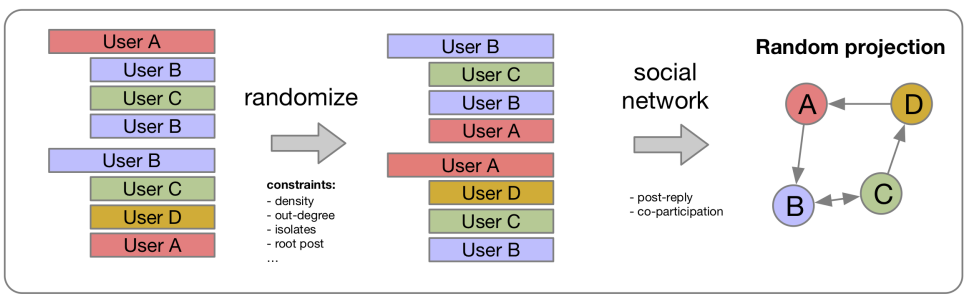

Figure 1: Comparison of network construction and randomization between modelling social networks (top) and forum-based modelling suggested in this study (bottom)

growth of these thread-trees is often modelled to also account for the parameters describing the temporal dynamics of forum participation.

\subsection{This Study's Approach to Null Models}

Figure 1 demonstrates the differences in creating null models for learner communication as derivative of forum-based modeling versus social modeling. Traditional methodology for social network analysis from forum data adopted in LA studies is captured in Figure 1 (top), and compared with our suggested approach (Figure 1, bottom). Forum posting activity can be viewed as a user-post network, where posts are included as nodes. User-post network can be projected into a social network, hereafter referred to as "observed projection". Traditional analyses, including in LA, then evaluate random graphs simulated from this projection of a social network to recapitulate various constraints (density, degree distribution, transitivity, reciprocity, and similar). However, this approach assumes that the derived social projection represents a bona fide social network. In this paper, we argue that social interactions are embedded in the original forum network (Figure 1, bottom). Depending on the structure of this original network (linked to the class-specific usage of the online tool), certain properties of the derived social network can be expected at random. In order to model the expected random social network, we simulate random graphs from the original forum network that constrain various features, such as network density, being a root post or an isolated post, or post popularity (out-degree). In other words, here we simulate null models from a post-network, not from observed learner-to-learner projection.

\subsection{Null Models Generation}

This study explores simple hypotheses about how online forum networks form. We simulate random graphs that represent posting activity (forum-based modelling approach, Figure 1, bottom). These random graphs are constrained around how many posts an individual makes (student posting activity) and how many replies a post receives (post popularity). These processes were selected as they representative of individual learner characteristics, rather than social dynamics.

In the first instance, we simulated forum communication as a tree-network of forum posts by different learners (Figure 2, left). This model tested two hypotheses: 1) Student activity (number of posts individual makes) explains how network forms. Here, random graph is generated controlling for posting activity through fixed density; and 2) The properties of the post explain how network forms. Here, random graphs are generated to control for post popularity through fixed out-degree.

In the second instance, forum communication was represented as a bipartite graph of learners posting into different discussion threads (Figure 2, right). Two hypotheses about network formation were tested: 1) Student activity (number of threads individual posts into) can explain how network forms. Here a random graph is simulated to control for posting activity through fixed learner out-degree (number of threads participated in); and 2) The properties of the thread 

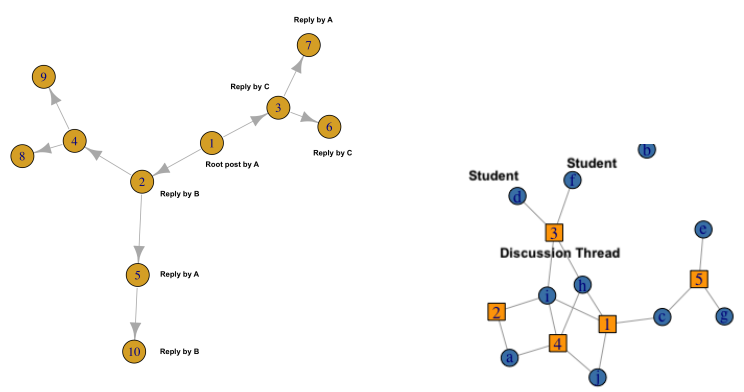

Figure 2: Null models simulated in the study for the comparison of their learner-to-learner projections to the observed real-life forum networks: post-to-post (left) and student-to-thread (right)

(number of learners participating in it) can explain how network forms. Random graphs control for discussion thread popularity through fixed in-degree (number of learners participating in it).

Simulated graphs are then projected into learner-to-learner weighted graphs (i.e. random projections) and compared to their observed projections. This yielded a total of six projections from two null models:

1) Weighted co-participation projection of a post-tree network controlling for student activity (i.e. tree_shared_activity);

2) Weighted post-reply projection (i.e. tree_answer_activity) of a post-tree network controlling for student activity;

3) Weighted co-participation projection of a post-tree network controlling for post popularity (i.e. tree_shared_topology);

4) Weighted post-reply projection of a post-tree network controlling for post popularity (i.e. tree_answer_topology);

5) Weighted co-participation projection of a student-to-thread network controlling for student activity (i.e. bi_stu-tostu_activity);

6) Weighted co-participation projection of a student-to-thread network controlling for thread popularity (i.e. bi_stu-tostu_topology).

\subsection{Details of Randomisation}

The randomization of edges in each of the nulls was implemented as follows. All forum posts (whether replied to or not) were included in the directed one-mode post-tree networks. Statnet R package was used to simulate random graphs using the formula specified in Table 1.

The tree_post activity model randomized which posts connected to which posts, as well as for the number of discussions within the network, as branches could contain different posts (and hence authors) than in the observed data. During the modelling, each post-node preserved author attributes, therefore, controlling for the volume of activity (number of posts) at the student level. The model constrained each post can only be given in response to one other post, as well as for the propensity of root node to have nodes to be attached to it.

The post_tree topology model controlled for the mechanisms described above, as well as preserved the out-degree of each post, therefore controlling for the popularity of post content. The model did not control which student chose to reply to which post, consequently, social processes such as homophily were excluded from the modelling.

For creating networks of the two-mode student-discussion nulls, $\mathrm{R}$ functions sample and birewire.rewire.bipartite were used to first shuffle the edges of one mode (students), and then to randomize edges for the second mode (discussions). First, when edges were randomized to control for activity only, students would randomly attach to discussion threads, i.e. who appeared with whom in the discussion thread was no longer controlled for. This model also did not constrain how much a given student posted, neither how much input a given discussion received. The second scenario for this null controlled both for student activity and discussion popularity, fixing degrees for both network modes. As the edges were randomly distributed from students to discussions, the model did not control which student chooses to post into which thread, so social processes like homophily were excluded.

\subsection{Comparing Observed Forum Networks to the Simulated Ones}

Each of these six null projections was compared to the observed data both in terms of global as well as local topological attributes. For each null model, twenty randomized networks were generated for the comparisons with the observed network. For each network (null and observed projections), we computed the following global topological features: 
Table 1: Mapping the student-to-student networks projected from thread-tree and student-to-thread null models, for comparisons with the observed networks. The labels of projections should be used to interpret visual results in Section 4.

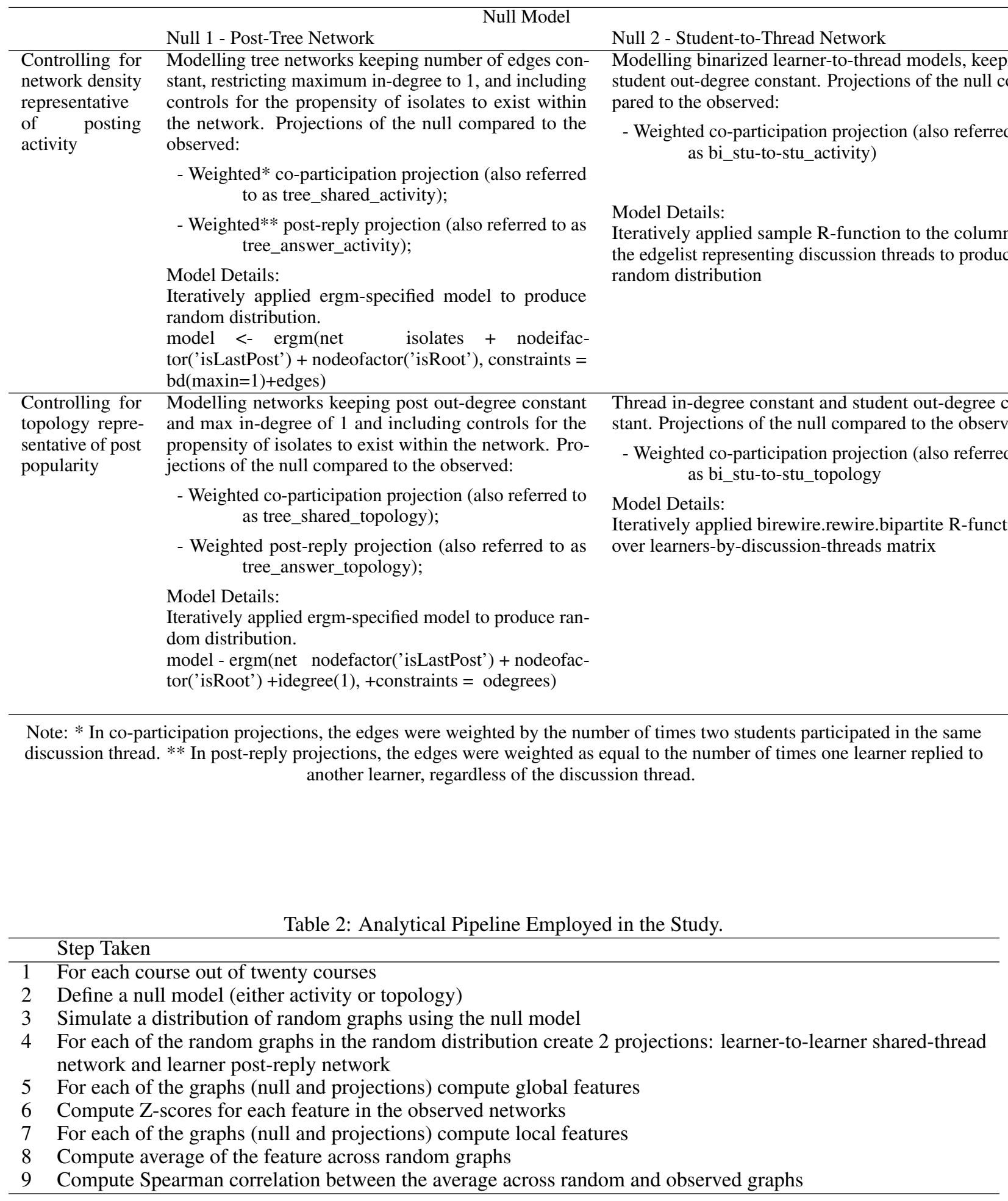


Table 3: Descriptive Statistics of Posting Activity in Online Forums.

\begin{tabular}{lllllllllllllllllllll}
\hline & A1 & B2 & C3 & D4 & E5 & F6 & G7 & H8 & I9 & J10 & K11 & L12 & M13 N14 & O15 & P16 & Q17 & R18 & S19 & T20 \\
\hline N_Students 40 & 25 & 35 & 68 & 133 & 85 & 22 & 35 & 41 & 23 & 20 & 202 & 30 & 87 & 27 & 47 & 74 & 135 & 515 & 49 \\
M_PPT* & 1.6 & 1.4 & 2 & 3.8 & 2.7 & 3.3 & 21 & 9.8 & 1.7 & 1.8 & 1.9 & 1.4 & 2.7 & 5.5 & 1.7 & 1.2 & 1.9 & 1.7 & 1.4 & 1.7 \\
N_Posts & 464 & 538 & 589 & 4257 & 2231 & 2339 & 560 & 655 & 719 & 346 & 388 & 2417 & 179 & 2304 & 818 & 1327 & 2392 & 2787 & 3368 & 369 \\
M_R & 0.4 & 0.3 & 0.5 & 0.7 & 0.6 & 0.7 & 0.95 & 0.9 & 0.4 & 0.5 & 0.5 & 0.3 & 0.6 & 0.8 & 0.4 & 0.2 & 0.5 & 0.4 & 0.3 & 0.4 \\
\hline \multicolumn{18}{c}{ Note: * M_PPT - mean number of posts per thread; ** M_R - mean replies. }
\end{tabular}

Table 4: Descriptive Statistics for Observed Learner Projections

\begin{tabular}{|c|c|c|c|c|c|c|c|c|c|c|c|c|c|c|c|c|c|}
\hline & & & & & $\mathrm{C} 3$ & $\mathrm{H} 8$ & $\mathrm{~A} 1$ & I9 & T20 & $\mathrm{D} 4$ & O17 & F6 & N14 & E5 & & 12 & \\
\hline & & 0.1 & 0.0 & 0.1 & 0.4 & 0.44 & 0.86 & 0. & $\begin{array}{ll}0.15 & 0.08\end{array}$ & 0.11 & & 0.46 & 0.33 & 0.2 & & & \\
\hline & & 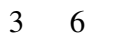 & 3 & & & & & & & 1 & & & & & & & \\
\hline & & & & & & & & & & & & 0.25 & & 0.23 & 53 & 67 & \\
\hline
\end{tabular}

number of communities (i.e. network modularity using infomap [33], network density, and the Gini coefficient for the network degree (i.e. as a measure of degree statistical dispersion). For each of these features, a Z-score of the feature was derived in the observed network compared to its value in the networks corresponding to a given null:

$$
\mathrm{Z}_{f n}=\frac{\chi_{f n}-\mu_{f n}}{\rho_{f n}}
$$

where $\chi_{f n}$ is the value of the feature $f$ in the observed network, and $\mu_{f n}$ and $\rho_{f n}$ are the mean and standard deviation of the feature value across the random networks under null model $n$. Null models for which $\left|Z_{f n}\right|<2$ are considered as explaining the feature $f$.

In the context of local, node-level features, we focus on the following metrics: weighted degree (strength), degree, subgraph centrality [33], leverage centrality [34], Burt's constraint [35], local clustering coefficient [36], and clustering weighted coefficient[37]. For each null, the average for each of these features was computed across all generated random networks. We then computed the Spearman correlation $\rho_{f n}$ between this average and the value obtained in the observed network. Such has enabled to quantify how well the null models captured the ranking of the node-level features in the observed networks. This process was conducted using data from all twenty courses. The entirety of our analytical pipeline is summarised in Table 2 .

\section{Results}

\subsection{Heterogeneity in Posting Activity}

The first research question inquired how much heterogeneity was observed in the posting activity across the different communication forums in twenty courses. Descriptive statistics at the posting activity level demonstrated high heterogeneity in forum participation. Table 3 describes the number of learners and posts in the forums, mean number of posts in the forum threads, as well as the mean number of replies.

The courses differed greatly in their pedagogical designs and forum strategies. The pedagogical variety help explain a large diversity in the level of posting activity and interactivity observed across the courses. Courses G7 and H8 provisioned a task of replying to 1-2 learners, as an assessed activity. Course D4 had learners assigned in small groups for collaborative tasks that were regularly graded. In Course N14, the instructor assessed individuals for solo posts. In this course, learners were asked to post individual reflections into discussion thread created for specific topics, which is why the number of posts in those threads appears higher. Otherwise, the number of posts per thread appears comparable across the courses, despite the highly heterogeneous total number of posts and learners.

Some courses appeared to have more balance in reciprocation, i.e. average number of replies. Again, the nature of tasks that elicited more individual posting or more interactive orientation for the discussions can help explain the differences. For instance, Course G7, where learners were graded in relation to their replies to build shared discourse, has a mean number of replies at 0.95 , i.e. almost every post received a reply. In contrast, for instance, course P16 had a mean of 0.2 posts reciprocated. This course had forum tasks that required to post individual-level reflections, and not so much interpersonal orientation. If approximated such dynamics implies that every eight out of ten posts were solo postings. The structure of discussion threads appears to help explain the length of threads here. In P16 the instructor 


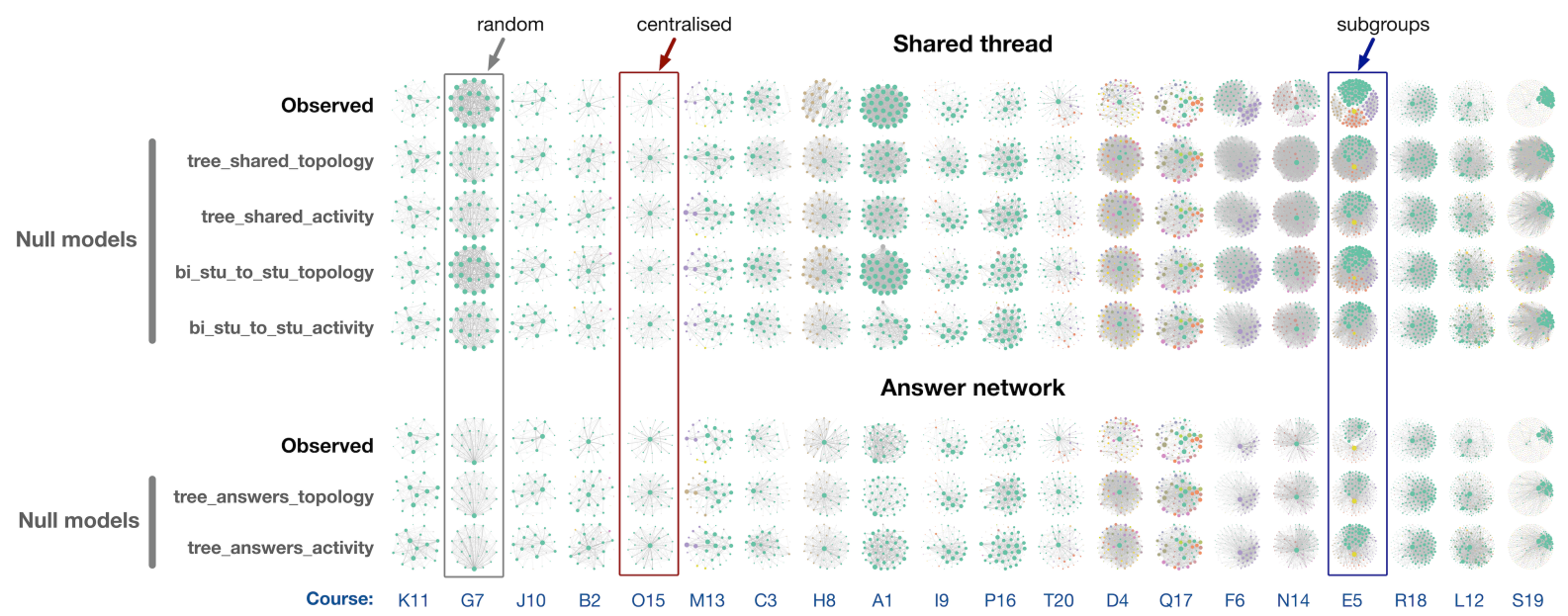

Figure 3: Visualisation of the networks obtained from the thread co-participation projection and the post-reply projections

a

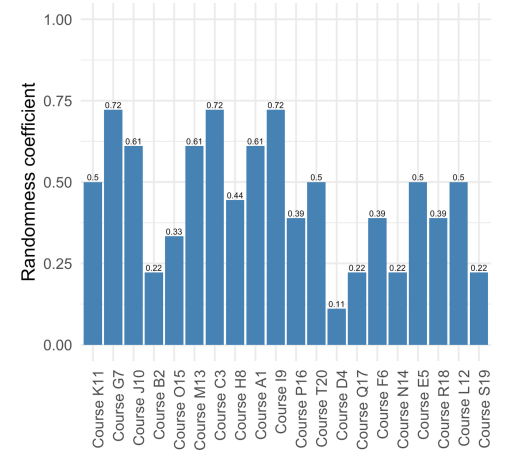

b

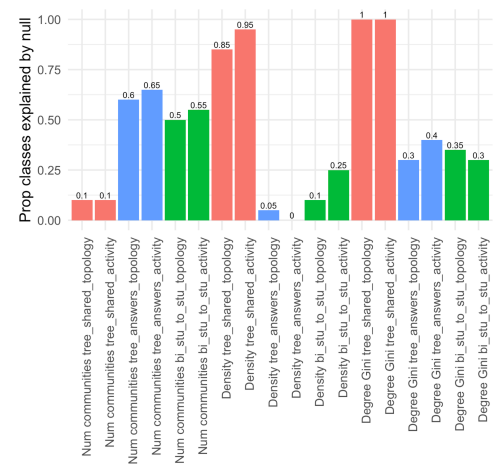

C

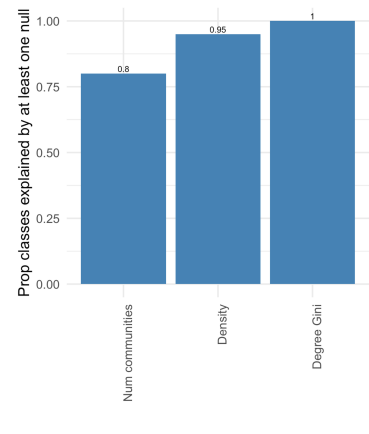

Figure 4: Comparison of observed and simulated network global metrics

structured the threads for individual posting that assigned a small number of groups to post in particular threads, and that is reflected in the length of the threads.

In conclusion, a high variability in the posting activity can to some extent be explained by the forum task design, suggesting that specific network structures are driven by what learners are asked to do. That is, unsurprisingly, the level of learner activity and its orientation is contingent on the design of the forum activities and course pedagogy.

\subsection{Understanding Observed vs Simulated Network Structures: Network-Level}

The second research question shifted the focus from posting activity towards the global network structures of network projections. It is non-trivial that learner posting depends on the forum task design. Yet, it is unclear if it is the posting activity that forms learner-to-learner network structures or social processes, as commonly hypothesised. To this end, we inquired how much of the student network (i.e. projections) global structure was explained by the posting behaviour. The global features compared across the random and observed projections were density and the number of communities. Figure 3 visualizes the global structures for the observed projections and their random projections (one random graph out of the distribution was selected for visualisation purposes). Figure 3 shows courses in the order of their size: courses with lower number of students - on the left, those with higher number of students - to the right. For each null model, we show one example random network out of the 20 generated. In the top row of Figure 3 we highlight three classes of network structures that can be found in the data. Course G7 appears to have a random structure. To explain, its random projections are similar in the global structure to the observed projections. This is the course where learners were required to post to any two other people, which appears to have occurred at random. Course O15 exemplifies communication with centralised structure, where nodes connected to one or few nodes, and not as much across one 
another. This course's random graphs also do not capture the centralised structure well. Due to the strong internship component in this course, the learners maintained dyadic interactions with instructor only, whereas would have had more connections with one another at random. Finally, course E5 captures the classroom with a clear community structure. We observe that random graphs do not reproduce the community formation (as such feature was not embedded within the null). Despite the differences for these courses with markedly different structures, for many courses random graphs reproduce projections that are rather similar to the observed projections.

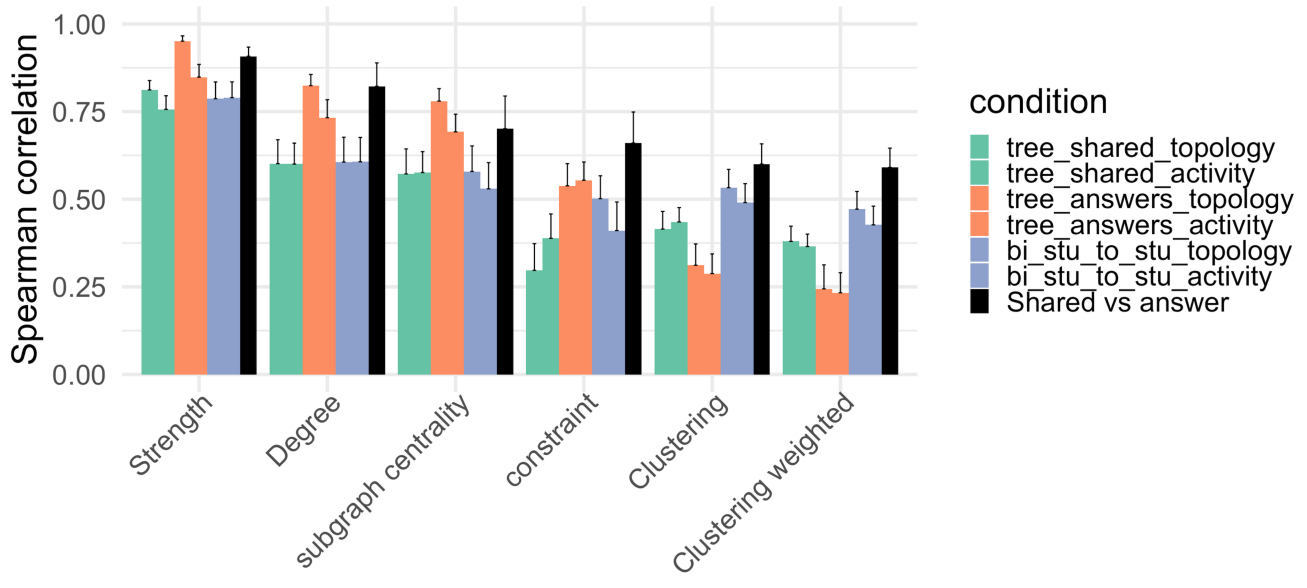

Figure 5: Comparison of observed and simulated network individual metrics

For the ease of interpretation, Table 4 contains descriptive statistics of the global network metrics that accompany Figure 3. Table 4 captures the number of communities per class, using the community detection method 'infomap' [32], network density for each of the courses, and the Gini coefficient of the degree for each of the courses. The Gini coefficient was used as an indicator of centralisation, where a higher Gini coefficient refers to a more unequal degree distribution. These descriptive statistics represent a diversity of network metrics across the projected learner communication networks. Overall, it appears that random graphs are more explanatory for some courses.

To understand how explainable are the simple null models built around posting activity and popularity, we quantified the coefficient to capture the extent that the observed network was captured by the random null. We defined this 'randomness coefficient' as the proportion of nulls that explain three global features (density, number of communities, and the Gini coefficient) observed (having $|Z|<2$ for the 18 quantities in Figure $4 b$ ). This helps conclude that course G7, highlighted in Figure 3, is indeed among the three courses with maximally random structure. Some classes like D4 are very non-random, as can also be observed in Figure 3. D4 is the class with a lot of subgroups, is less dense, and more centralised than its random representations. In other words, different courses vary in terms of their 'randomness coefficient', and some are easier explained by the simple null models that control for learner activity and post popularity.

Similarly, the nulls we explored were not equal in their ability to capture features across different forum communication networks. For each null, we computed the proportion of courses where the feature in the observed network was well explained by random networks (see Methods). We see that the null models of student-to-student activity based on co-participation in the thread projected from post-reply networks (controlling for student activity and post popularity) explain well the density and Gini coefficient of the degree, but not the number of communities. On the other hand, null models of post-reply networks from tree-post networks and both co-participation and post-reply projections from bipartite networks explain well the number of communities but not network density and Degree Gini. Yet, overall, for most courses, at least one null model could explain one feature well.

To conclude, it appears that network structures in some courses are more random than in others; as well as some nulls are better at identifying certain features than others (e.g. communities of density). It should be noted that there is always a null model capturing some elements of the structure. That is, the level of student activity and the properties of the posts to a large extent can explain the structure of communication networks. 


\subsection{Understanding Observed vs Simulated Network Structures: Node Level}

Having examined forum posting activity and global network features, we focused on how well null models can capture node-level metrics, such as degree and strength. We asked what individual topological features were not derivative of online posting behavior.

We found that learner degree and weighted degree, both favoured by LA community to capture outcomes of social learning, to a large extent are explained by the null models. Figure 5 presents the Spearman correlation between network centralities in the nulls versus the observed network. A black bar in the plots represents the Spearman correlation between the observed shared-thread projection and the post-reply one. Both strength and degree measures have been found to have the highest correlation between projections and between observed and null networks. That is, null models that control for individual posting activity as well as the quality and relevance of post content are well captured by random graphs. Learner levels of activity may result from learner motivation or design impacting how much one posts. Post properties may relate to learner communication skills or ability to share relevant information. These are however proxies of individual competence to engage or communicate, not necessarily group-related benefits of social learning, although in LA degree and strength are often used to capture the latter.

In contrast, clustering, both weighted and non-weighted, is poorly described by nulls (mostly $<50 \%$ ). Within the current results clustering remains an 'independent' feature that could capture individual positioning within social learning opportunities - in contrast to the degree and its weight that appear to be largely driven by individual learner characteristics.

In sum, node-level metrics such as strength and degree are well captured by null models controlling for posting activity, whereas triadic metrics, such as Burt's constraint and clustering are not. This suggests that higher-order social phenomena are not well presented by the simple null models. Student-to-student projections based on thread co-participation projected from bipartite nulls perform best in capturing triadic-level features.

\section{Discussion}

We have argued that existing analyses of learner interactions in digital learning environments in LA are limited. The association between network indicators at the global level, such as density, and at the local level, such as learner degree, are commonly examined in relation to learner performance, however, it remains unclear whether such observed associations represent bona fide social effects, or merely reflect heterogeneity in posting activity. To address this issue, the study has applied various null models that include basic generative processes for posting activity, to evaluate networks of forum communication. Through the application of these null models, the study examined which features of student network topology are mere derivatives of upstream forum processes.

The study makes several contributions to the future research in the area of network analytical applications in LA. First, we detail the methodology that can be replicated to further examine other generative processes. Specifically, in this study we generated null models (tree-post networks and bipartite student-to-thread networks) to control for student activity and content popularity at the post-level and discussion-thread levels. Within the methodology, these were further mapped to learner-to-learner projections of the nulls, i.e. post-reply and learner-to-thread networks, to then test further hypothesis using null models. Observed and random projections were compared using statistical approaches. In particular, we distinguished the null models based on the properties such as node attributes, the way how the activity of a learner (student) is encrypted and what is preserved during edge randomization process.

Besides the methodology, our empirical results can form a foundation for the future empirical analyses in networks. Among the challenges noted during the study is that the online forum communication networks in learning settings are heterogeneous, with diversity in group sizes, number of posts per course, varying activity level across learners, and subgroup formation. Still, using our methodology we could identify how much of the student network global structure and local node-level metrics were explained by the online forum posting behaviour. The structures underpinning projected learner interactions were diverse, but when compared to the null models, some structures appeared more random than others. Network density, for instance, to a large extent can be reproduced by simple models that control for posting activity and content popularity. Number of communities are not easily reproduced using the two variations of nulls we explored. We find that across all courses, there is almost always one null model that describes the data well. In terms of the local network measures capturing learner-level network metrics, we found that 'ego' metrics such as strength and degree could be well captured by the null models, whereas triadic metrics, such as Burt's constraint or clustering, were not.

Our results emphasize that individual-level posting behaviour and its properties are among fundamental processes that form the structure of communication networks. This has several implications. First, posting activity needs to be integrated into the generative processes of network formation, with the volume of posts controlled for in the models. 
Second, using null models of posting activity can be used to uncover forum structures that appear more random. Comparing network-level features across random and observed graphs would allow for cross-course evaluations - an otherwise challenging task given the diversity of metrics across the forums.

Finally, the results of the study caution against the use of certain global and local network measures to capture interpersonal engagement in social learning. Both network density and node-level measures, similar to the ones analysed, are commonly used in LA to capture social learning outcomes. Yet, we show that individual level measures, such as degree or frequency of dyadic interactions reflect individual learning activity, i.e. behaviours directed towards the group by an individual. Hence, such metrics capture contributions to the social participation. These can be evaluated as a part of social learning activities. Instructors can assess if their tasks are prompted higher or lower learner activity levels. Measures other than degree or edge weight are needed to capture the effect of social dynamics or networked activity. Our empirical investigation based on simple assumptions about posting activity shows that the number of communities or such local features as clustering may be among measures.

It should be noted that among important limitations of the work presented is that our methodology did not account explicitly for the existence of subgroups (or communities). This limitation could be solved through the use of stochastic block models (SBM) [38]. SBM enables to generate graphs containing communities that are connected with one another through particular edge densities; these null models can shed light unto network structures that posting activity nulls described in the study could not capture.

Future work should focus on the theoretical elaboration of the generative processes and consequent temporal modelling of forum communication formation. Developing such models would be most aligned with current network scientific views on how similar activity-driven networks form. Researchers could also compare the structures of the reconstructed learner-to-learner networks with the additional level of existing interactions, i.e. either self-reports or existing trace data. Alternatively, the level of complexity to the simple nulls can be added by understanding the role of node attributes such as status (instructor, TA), age, grade, or other types of links (i.e. interpersonal relationships) to test statistically how these parameters influence the learning outcomes.

\section{Acknowledgements}

We thank three anonymous reviewers and a meta-reviewer of the LAK'20 Program Committee, as well as Shane Dawson and Bodong Chen for their critical feedback on this paper.

\section{References}

[1] Shane Dawson, Srecko Joksimovic, Oleksandra Poquet, and George Siemens. Increasing the impact of learning analytics. In Proceedings of the 9th International Conference on Learning Analytics \& Knowledge, LAK19, pages 446-455, New York, NY, USA, 2019. ACM.

[2] Rebecca Ferguson, Andrew Brasher, Doug Clow, Adam Cooper, Garron Hillaire, Jenna Mittelmeier, Bart Rienties, Thomas Ullmann, and Riina Vuorikari. Research evidence on the use of learning analytics: Implications for education policy. 2016.

[3] Jennifer P. L. Tan, Elizabeth Koh, Christin R Jonathan, and Simon Yang. Learner dashboards a double edged sword? students' sense-making of a collaborative critical reading and learning analytics environment for fostering 21 st century literacies. The Journal of Learning Analytics, 4(1), 2017.

[4] Jian-Syuan Wong and Xiaolong Zhang. MessageLens: A visual analytics system to support multifaceted exploration of MOOC forum discussions. Visual Informatics, 2(1):37-49, 2018.

[5] Marielle Dado and Daniel Bodemer. A review of methodological applications of social network analysis in computer-supported collaborative learning. Educational Research Review, 22(Supplement C):159-180, 2017.

[6] Xueye Chen, Bo Liu, Jingtao Zhu, Mu Gu, Hong Chen, Jinliang Liu, Liang Chen, and Xiaoping Ouyang. Light extraction enhancement and directional control of scintillator by using microlens arrays, 2018 .

[7] Mina Shirvani Boroujeni and Pierre Dillenbourg. Discovery and temporal analysis of latent study patterns in MOOC interaction sequences. In Proceedings of the 8th International Conference on Learning Analytics and Knowledge, LAK'18, pages 206-215, New York, NY, USA, 2018. ACM.

[8] A F Wise, Y Cui, and W Q Jin. Honing in on social learning networks in MOOC forums: examining critical network definition decisions. In Proceedings of the 7th International Conference for Learning Analytics and Knowledge, 2017. 
[9] Dan Suthers. From contingencies to network-level phenomena: Multilevel analysis of activity and actors in heterogeneous networked learning environments. In Proceedings of the Fifth International Conference on Learning Analytics And Knowledge, LAK' 15, pages 368-377, New York, NY, USA, 2015. ACM.

[10] Carmel Kent, Amit Rechavi, and Sheizaf Rafaeli. Networked learning analytics: A theoretically informed methodology for analytics of collaborative learning. In Yael Kali, Ayelet Baram-Tsabari, and Amit M Schejter, editors, Learning In a Networked Society: Spontaneous and Designed Technology Enhanced Learning Communities, pages 145-175. Springer International Publishing, Cham, 2019.

[11] Adrienne Traxler, A Gavrin, and Rebecca Lindell. Networks identify productive forum discussions. Phys. Rev. Phys. Educ. Res., 14(2):020107, 2018.

[12] Justyna P Zwolak, Remy Dou, Eric A Williams, and Eric Brewe. Students' network integration as a predictor of persistence in introductory physics courses. Phys. Rev. Phys. Educ. Res., 13(1):010113, 2017.

[13] Srećko Joksimović, Areti Manataki, Dragan Gašević, Shane Dawson, Vitomir Kovanović, and Inés Friss de Kereki. Translating network position into performance: Importance of centrality in different network configurations. In Proceedings of the Sixth International Conference on Learning Analytics \& Knowledge, LAK '16, pages 314-323, New York, NY, USA, 2016. ACM.

[14] Shazia K Jan and Panos Vlachopoulos. Influence of learning design of the formation of online communities of learning. International Review of Research in Open and Distributed Learning, 19(4), 2018.

[15] Pablo Aragón, Vicenç Gómez, David García, and Andreas Kaltenbrunner. Generative models of online discussion threads: state of the art and research challenges. Journal of Internet Services and Applications, 8(1):15, 2017.

[16] Eugene Borokhovski, Robert M Bernard, Rana M Tamim, Richard F Schmid, and Anna Sokolovskaya. Technologysupported student interaction in post-secondary education: A meta-analysis of designed versus contextual treatments. Computers Educatio, 96:15-28, 2016.

[17] Leah P Macfadyen and Shane Dawson. Mining LMS data to develop an "early warning system" for educators: A proof of concept. Computers Education, 54(2):588-599, 2010.

[18] Srećko Joksimović, Oleksandra Poquet, Vitomir Kovanović, Nia Dowell, Caitlin Mills, Dragan Gašević, Shane Dawson, Arthur C Graesser, and Christopher Brooks. How do we model learning at scale? a systematic review of research on MOOCs. Review of Educational Research, 88(1):43-86, 2018.

[19] Ed Fincham, Alexander Whitelock-Wainwright, Vitomir Kovanović, Srećko Joksimović, Jan-Paul van Staalduinen, and Dragan Gašević. Counting clicks is not enough: Validating a theorized model of engagement in learning analytics. In Proceedings of the 9th International Conference on Learning Analytics \& Knowledge, LAK19, pages 501-510, New York, NY, USA, 2019. ACM.

[20] René F Kizilcec and Emily Schneider. Motivation as a lens to understand online learners: Toward Data-Driven design with the OLEI scale. ACM Transactions on Computer-Human Interaction (TOCHI), 22(2):6:1-6:24, 2015.

[21] Ed Fincham, Dragan Gašević, and Abelardo Pardo. From social ties to network processes: Do tie definitions matter? Journal of Learning Analytics, 5(2):9-28-9-28, 2018.

[22] Urlich Hoppe. Computational methods for the analysis of learning and knowledge building communities. In Charles Lang, George Siemens, Alyssa Wise, and Dragan Gasevic, editors, Handbook of Learning Analytics, pages 23-33. Society for Learning Analytics Research (SoLAR), first edition, 2017.

[23] Dean Lusher, Johan Koskinen, and Garry Robins. Exponential Random Graph Models for Social Networks: Theory, Methods, and Applications. Cambridge University Press, 2013.

[24] Shaun Kellogg, Sherry Booth, and Kevin Oliver. A social network perspective on peer supported learning in MOOCs for educators. The International Review of Research in Open and Distributed Learning, 15(5), 2014.

[25] Jingjing Zhang, Maxim Skryabin, and Xiongwei Song. Understanding the dynamics of MOOC discussion forums with simulation investigation for empirical network analysis (SIENA). Distance Education, 37(3):270-286, 2016.

[26] Thushari Atapattu, Katrina Falkner, and Hamid Tarmazdi. Topic-Wise classification of MOOC discussions: A visual analytics approach. International Educational Data Mining Society, 2016.

[27] Farshid Marbouti and Alyssa Friend Wise. Starburst: a new graphical interface to support purposeful attention to others' posts in online discussions. Educational Technology Research and Development, 64(1):87-113, 2016.

[28] M Ángeles Serrano, Marián Boguñá, and Alessandro Vespignani. Extracting the multiscale backbone of complex weighted networks. PNAS, 106(16), 2009.

[29] Mark Newman. Networks. Oxford University Press, 2018. 
[30] Subhayan Mukerjee, Tian Yang, and Sandra González-Bailón. What counts as a weak tie? a comparison of filtering techniques for weighted networks. 2019.

[31] Mark S Granovetter. The strength of weak ties. In Social networks, pages 347-367. Elsevier, 1977.

[32] Alexey N Medvedev, Renaud Lambiotte, and Jean-Charles Delvenne. The anatomy of reddit: An overview of academic research. In Dynamics on and of Complex Networks, pages 183-204, 2017.

[33] Martin Rosvall and Carl T Bergstrom. Maps of information flow reveal community structure in complex networks. arXiv preprint physics. soc-ph/0707. 0609, 2007.

[34] Karen E Joyce, Paul J Laurienti, Jonathan H Burdette, and Satoru Hayasaka. A new measure of centrality for brain networks. PLoS One, 5(8):e12200, 2010.

[35] Ronald Burt. Structural holes. cambridge, MA, 1992.

[36] D J Watts and S H Strogatz. Collective dynamics of 'small-world' networks. Nature, 393(6684):440-442, 1998.

[37] A Barat, M Barthelemy, and A Vespignani. Weighted evolving networks: coupling topology and weighted dynamics. Phys. Rev. Lett., 92(22):228701, 2004.

[38] Tiago P Peixoto. Hierarchical block structures and High-Resolution model selection in large networks. Phys. Rev. $X, 4(1): 011047,2014$. 